\title{
Analysis of a class of nonlinear fractional differential models generated by impulsive effects
}

\author{
Wenbin Liu ${ }^{1 *}$, Mengqiu Wang ${ }^{2}$ and Tengfei Shen ${ }^{1}$
}

\section{"Correspondence:}

liuwenbin-xz@163.com

'School of Mathematics, China

University of Mining and

Technology, Xuzhou, 221116,

P.R. China

Full list of author information is

available at the end of the article

\begin{abstract}
The purpose of this paper is to analyze a class of nonlinear fractional differential models generated by impulsive effects. Based on variational methods, some new results are obtained in regard to the existence of solutions. Moreover, our results enrich and extend some existing conclusions.
\end{abstract}

MSC: 26A33; 34G20; 34B15

Keywords: fractional applied differential model; impulsive effect; boundary value problem; $p$-Laplacian operator

\section{Introduction}

In this paper, we investigate the existence of solutions for an impulsive fractional Dirichlet boundary value problem with a $p$-Laplacian operator and a controlled parameter as follows:

$$
\left\{\begin{array}{l}
{ }_{t} D_{T}^{\alpha} \phi_{p}\left({ }_{0} D_{t}^{\alpha} u(t)\right)+a(t) u(t)=f(t, u(t))+\mu b(t)|u(t)|^{\nu-2} u(t), \quad \text { a.e. } t \in J, \\
\Delta\left({ }_{t} I_{T}^{1-\alpha} \phi_{p}\left({ }_{0} D_{t}^{\alpha} u\left(t_{j}\right)\right)\right)=I_{j}\left(u\left(t_{j}\right)\right), \quad j=1,2, \ldots, m, m \in \mathbb{N} \\
u(0)=u(T)=0
\end{array}\right.
$$

where $a(t), b(t) \in C([0, T], \mathbb{R}) ; f(t, u) \in C([0, T] \times \mathbb{R}, \mathbb{R}) ; \mu \in(0, \infty), p \in(1, \infty), \alpha \in\left[\frac{1}{p}, 1\right)$, $v \in[1, p-1) ; t_{0}=0<t_{1}<t_{2}<\cdots<t_{m}<t_{m+1}=T ; J=[0, T] \backslash\left\{t_{1}, t_{2}, \ldots, t_{m}\right\} ; \phi_{p}(s)=|s|^{p-2} s$ $(s \neq 0), \phi_{p}(0)=0 ; I_{j} \in C(\mathbb{R}, \mathbb{R})$;

$$
\begin{aligned}
& \Delta\left({ }_{t} I_{T}^{1-\alpha} \phi_{p}\left({ }_{0}^{c} D_{t}^{\alpha} u\left(t_{j}\right)\right)\right)={ }_{t} I_{T}{ }^{1-\alpha} \phi_{p}\left({ }_{0}^{c} D_{t}^{\alpha} u\left(t_{j}^{+}\right)\right)-{ }_{t} I_{T}{ }^{1-\alpha} \phi_{p}\left({ }_{0}^{c} D_{t}^{\alpha} u\left(t_{j}^{-}\right)\right), \\
& { }_{t} I_{T}{ }^{1-\alpha} \phi_{p}\left({ }_{0}^{c} D_{t}^{\alpha} u\left(t_{j}^{+}\right)\right)=\lim _{t \rightarrow t_{j}^{+}} I_{T}^{1-\alpha} \phi_{p}\left({ }_{0}^{c} D_{t}^{\alpha} u\left(t_{j}\right)\right), \\
& { }_{t} I_{T}{ }^{1-\alpha} \phi_{p}\left({ }_{0}^{c} D_{t}^{\alpha} u\left(t_{j}^{+}\right)\right)=\lim _{t \rightarrow t_{j}^{-}} I_{T}^{1-\alpha} \phi_{p}\left({ }_{0}^{c} D_{t}^{\alpha} u\left(t_{j}\right)\right) .
\end{aligned}
$$

In the recent years, more and more fractional mathematical models have occurred in many application fields (see [1-4]). It should be pointed out that Leszczynski and Blaszczyk [5] took advantage of the fractional mathematical model to show that the height of granular

(c) The Author(s) 2017. This article is distributed under the terms of the Creative Commons Attribution 4.0 International License (http://creativecommons.org/licenses/by/4.0/), which permits unrestricted use, distribution, and reproduction in any medium, provided you give appropriate credit to the original author(s) and the source, provide a link to the Creative Commons license, and indicate if changes were made. 
material of silo decreases over time as follows.

$$
{ }_{t}^{c} D_{T}^{\alpha}\left({ }_{0} D_{t}^{\alpha} h^{*}(t)\right)+\beta h^{*}(t)=0, \quad t \in[0, T],
$$

where $\alpha \in(0,1),{ }_{t}^{c} D_{T}^{\alpha}$ represents the right Caputo fractional derivatives, ${ }_{0} D_{t}^{\alpha}$ denotes left Riemann-Liouville fractional derivatives, $h^{*}(t)=h_{\text {bed }}-h(t)$ in which $h(t)$ and $h_{\text {bed }}$ stand for falling height of the granular bed relative to empty silo and the initial bed height, respectively.

Some classical nonlinear functional methods have been applied to investigate solvability of boundary value problems for fractional differential equations such as fixed point theorems (see [6-9]), coincidence degree theory (see [10-12]), etc. Recently, the operator ${ }_{t}^{c} D_{T}^{\alpha}{ }_{0} D_{t}^{\alpha}$ with the classical Dirichlet boundary condition possessing variational structure has been presented in [13], in which the following fractional differential system was considered:

$$
\left\{\begin{array}{l}
{ }_{t} D_{T}^{\alpha}\left({ }_{0} D_{t}^{\alpha} u(t)\right)=\nabla F(t, u(t)), \quad \text { a.e. } t \in[0, T], \\
u(0)=u(T)=0
\end{array}\right.
$$

where $\alpha \in(0,1] ; F(t, u):[0, T] \times \mathbb{R}^{N} \rightarrow \mathbb{R}$ satisfies the classical Carathéodory condition. It should be mentioned that ${ }_{t} D_{T}^{\alpha} u={ }_{t}^{c} D_{T}^{\alpha}$ and ${ }_{0} D_{t}^{\alpha} u={ }_{0}^{c} D_{t}^{\alpha}$ when $u(0)=u(T)=0$. Based on the mountain pass theorem and under $F(t, u)$ satisfying a class of superlinear growth conditions and the classical local Ambrosetti-Rabinowitz condition

$$
0<F(t, u) \leq \frac{1}{\theta}\langle u, \nabla F(t, u)\rangle, \quad \theta>2,|u| \geq M \text { or } u \in \mathbb{R} \backslash\{0\},
$$

where $\theta, M>0$ are constants, the existence of weak nontrivial solutions was proved when $\alpha \in\left(\frac{1}{2}, 1\right]$. In fact, if $\alpha \in\left(\frac{1}{2}, 1\right]$, the so-called fractional Sobolev space $E_{0}^{\alpha, 2}$ is compactly embedded into $C([0, T], \mathbb{R})$. Thus, the proof is more clear. Torres [14] gave the further study on this type problem when $\mathbb{N}=1$. Chen and Liu [15] extended the corresponding results on (1.3) to a $p$-Laplacian operator case where $1<p<\infty$. Moreover, Bergounioux et al. [16], Idczak and Walczak [17] and Jin and Liu [18] devoted their works to perfecting a fractional-type Sobolev space. It should be pointed out that Bonanno et al. [19] investigated the following impulsive fractional Dirichlet boundary value problem:

$$
\left\{\begin{array}{l}
{ }_{t} D_{T}^{\alpha}\left({ }_{0}^{c} D_{t}^{\alpha} u(t)\right)+a(t) u(t)=\lambda f(t, u(t)), \quad \text { a.e. } t \in J, \\
\Delta\left({ }_{t} I_{T}^{1-\alpha}\left({ }_{0}^{c} D_{t}^{\alpha} u\left(t_{j}\right)\right)\right)=\mu I_{j}\left(u\left(t_{j}\right)\right), \quad j=1,2, \ldots, m, \\
u(0)=u(T)=0,
\end{array}\right.
$$

where $a(t) \in C([0, T],(0, \infty)), f(t, u) \in C([0, T] \times \mathbb{R}, \mathbb{R})$ and $\lambda, \mu \in(0, \infty)$, and proved the existence of three solutions. For application of variational methods in boundary value problems of integer or fractional differential equations with impulsive effects, please see [20-27] and the references therein.

After that, by the gene property, Ledesma and Nyamoradi [28] studied the following eigenvalue problem:

$$
\left\{\begin{array}{l}
{ }_{t} D_{T}^{\alpha} \phi_{p}\left({ }_{0} D_{t}^{\alpha} u(t)\right)=\lambda \phi_{p}(u), \quad t \in[0, T], \\
u(0)=u(T)=0
\end{array}\right.
$$


and found that $\Lambda:=\{\lambda \in \mathbb{R}: \lambda$ is an eigenvalue of (1.5) $\}$ is a nonempty infinite set, $\sup \Lambda=$ $\infty$ and

$$
\lambda_{1}=\inf _{u \in E_{0}^{\alpha, p} \backslash\{0\}} \frac{\left.\left.\int_{0}^{T}\right|_{0} D_{t}^{\alpha} u(t)\right|^{p} d t}{\int_{0}^{T}|u(t)|^{p} d t}>0 .
$$

Moreover, in [28], based on the above results, the authors took further study of the following impulsive fractional Dirichlet boundary value problem with $p$-Laplacian operator:

$$
\left\{\begin{array}{l}
{ }_{t} D_{T}^{\alpha} \phi_{p}\left({ }_{0} D_{t}^{\alpha} u(t)\right)+a(t) u(t)=f(t, u(t)), \quad \text { a.e. } t \in J, \\
\Delta\left({ }_{t} I_{T}^{-\alpha} \phi_{p}\left({ }_{0} D_{t}^{\alpha} u\left(t_{j}\right)\right)\right)=I_{j}\left(u\left(t_{j}\right)\right), \quad j=1,2, \ldots, m, \\
u(0)=u(T)=0 .
\end{array}\right.
$$

For stating the main results of [28], we firstly introduce the following assumptions with respect to $f$ and $I_{j}$ :

(H0) $a(t) \in C([0, T], \mathbb{R})$ and $\operatorname{essinf}_{t \in[0, T]} a(t)>-\lambda_{1}$.

(H1) $f(t, u)=o\left(|u|^{p-1}\right)$ as $|u| \rightarrow 0$ uniformly for $t \in[0, T]$.

(H2) There exist constants $D>0, \theta>p$ such that, for $t \in[0, T]$,

$$
0<\theta F(t, u) \leq u f(t, u) \quad \text { for }|u| \geq D,
$$

where $F(t, u)=\int_{0}^{u} f(t, s) d s$.

(H3) There exist constants $d_{j}>0$ and $\gamma_{j} \in(p-1, \theta-1)$,

$$
\left|I_{j}(t)\right| \leq d_{j}|t|^{\gamma_{j}} \quad \text { for any } t \in \mathbb{R} \text {. }
$$

(H4) For $t$ large enough, $I_{j}(t)$ satisfy

$$
\theta \int_{0}^{t} I_{j}(s) d s \geq I_{j}(t) t
$$

(H5) For any $t \in \mathbb{R}, I_{j}(t)$ satisfy

$$
\int_{0}^{t} I_{j}(s) d t \geq 0
$$

(H6) $I_{j}(u)$ and $f(t, u)$ are odd on $u$.

Theorem 1.1 (see [28]) Assume that conditions (H0)-(H6) hold. Then problem (1.7) has infinitely many weak solutions.

Motivated by the works mentioned above, we take further study on this topic with the concave-convex nonlinearity (1.1). For comparing our main result with Theorem 1.1, we present the following assumed conditions and our main result.

$\left(\mathrm{H} 2^{\prime}\right)$ There exist constants $D>0, \theta>p$ such that, for $t \in[0, T]$,

$$
\theta F(t, u) \leq u f(t, u) \quad \text { for }|u| \geq D
$$


and

$$
\inf _{|u|=D} F(t, u)>0
$$

$\left(\mathrm{H}^{\prime}\right)$ There exist constants $a_{j}, d_{j}>0$ and $\gamma_{j} \in[0, \theta-1)$

$$
\left|I_{j}(t)\right| \leq a_{j}+d_{j}|t|^{\gamma_{j}} \quad \text { for any } t \in \mathbb{R}
$$

(H7) There exist constants $M, L>0, \beta>\theta$ such that, for $t \in[0, T]$,

$$
F(t, u) \leq M|u|^{\beta} \quad \text { for }|u| \geq L
$$

Now, we describe our main result.

Theorem 1.2 If conditions $(\mathrm{H} 0),(\mathrm{H} 1),\left(\mathrm{H} 2^{\prime}\right),\left(\mathrm{H}^{\prime}\right)$ and $(\mathrm{H} 4)-(\mathrm{H} 7)$ are satisfied, there exists a constant $\mu_{*}>0$ such that problem (1.1) has infinitely many weak solutions for $\mu \in\left[0, \mu_{*}\right)$.

Remark 1.3 It should be noted that $\left(\mathrm{H}_{2}^{\prime}\right)$ occurred earlier in [29] and was used to deal with multiplicity of solutions of fourth-order elliptic equations. Clearly, $\left(\mathrm{H} 2^{\prime}\right)$ and (H3') are weaker than (H2) and (H3), respectively. Moreover, if $\mu=0$, (H7) can be removed. Thus, our conclusion extends Theorem 1.1. Furthermore, if $\gamma_{j}$ is located in $\in[0, p-1)$ in $\left(\mathrm{H}^{\prime}\right)$, (H4) can be removed. Noting that if $p=2, \alpha=1$, one has

$$
{ }_{t} D_{T}^{\alpha}\left(\left|{ }_{0} D_{t}^{\alpha} u(t)\right|^{p-2}{ }_{0} D_{t}^{\alpha} u(t)\right)={ }_{t} D_{T}^{\alpha}\left({ }_{0} D_{t}^{\alpha} u(t)\right)=-u^{\prime \prime}
$$

Therefore, our main result also generalizes the corresponding result of [30].

Remark 1.4 Here, $I_{j}(u)$ could be $p$-suplinear or $p$-sublinear growth. It becomes more general than the previous papers (see [28] and [30]).

Moreover, we also consider the nonlinearity satisfying $p$-sublinear growth. We need the following assumptions.

$\left(\mathrm{H}^{\prime \prime}\right)$ There exist constants $a_{j}, d_{j}>0$ and $\gamma_{j} \in[0, p-1)$

$$
\left|I_{j}(t)\right| \leq a_{j}+d_{j}|t|^{\gamma_{j}} \quad \text { for any } t \in \mathbb{R}
$$

(H8) There exists a function $v \in E_{0}^{\alpha, p}$ such that

$$
\frac{1}{\mu} \int_{0}^{T} F(t, v(t)) d t+\frac{1}{v} \int_{0}^{T} b(t)|v(t)|^{v} d t>0 .
$$

$$
\limsup _{|u| \rightarrow \infty} \frac{F(t, u)}{|u|^{p}}=0, \quad \text { uniformly for } t \in[0, T]
$$

Theorem 1.5 If conditions (H0), (H2"), (H8) and (H9) are satisfied, there exists a constant $\mu^{*}>0$ such that problem (1.1) has at least one weak solution for $\mu \in\left(\mu^{*}, \infty\right)$. 


\section{Preliminaries}

First, we show the basic definitions and propositions of fractional integral and derivative, fractional-type Sobolev space. Let $\|u\|_{L^{p}}=\left(\int_{0}^{T}|u(t)|^{p} d t\right)^{\frac{1}{p}}$ and $\|u\|_{\infty}=\max _{t \in[0, T]}|u(t)|$ be the norms in $L^{p}([0, T], \mathbb{R})$ and $C([0, T], \mathbb{R})$, respectively. The constant $c$ stands for a different constant in different sentences.

Definition 2.1 ([2,3]) Let $f$ be a function defined on $[a, b]$.

(i) The left and right Riemann-Liouville fractional integrals of order $\alpha>0$ for a function $f$ are defined by

$$
\begin{aligned}
& { }_{a} I_{t}^{\alpha} f(t)=\frac{1}{\Gamma(\alpha)} \int_{a}^{t}(t-s)^{\alpha-1} f(s) d s, \\
& { }_{t} I_{b}^{\alpha} f(t)=\frac{1}{\Gamma(\alpha)} \int_{t}^{b}(s-t)^{\alpha-1} f(s) d s, \quad t \in[a, b],
\end{aligned}
$$

provided the right-hand sides are pointwise defined on $[a, b]$, where $\Gamma(\alpha)$ is the standard gamma function.

(ii) If $\alpha=n, n \in \mathbb{N}$, they become the usual definitions

$$
\begin{aligned}
& { }_{a} I_{t}^{n} f(t)=\frac{1}{\Gamma(n)} \int_{a}^{t}(t-s)^{n-1} f(s) d s, \\
& { }_{t} I_{b}^{n} f(t)=\frac{1}{\Gamma(n)} \int_{t}^{b}(s-t)^{n-1} f(s) d s, \quad t \in[a, b] .
\end{aligned}
$$

Definition $2.2([2,3])$ Let $f$ be a function defined on $[a, b]$.

(i) The left and right Riemann-Liouville fractional derivatives of order $\alpha$ for a function $f$ denoted by ${ }_{a} D_{t}^{\alpha} f(t)$ and ${ }_{t} D_{b}^{\alpha} f(t)$, respectively, are defined by

$$
{ }_{a} D_{t}^{\alpha} f(t)=\frac{d^{n}}{d t^{n}} I_{t}^{n-\alpha} f(t), \quad{ }_{t} D_{b}^{\alpha} f(t)=(-1)^{n} \frac{d^{n}}{d t^{t}} I_{b}^{n-\alpha} f(t),
$$

where $t \in[a, b], n-1 \leq \alpha<n$ and $n \in \mathbb{N}$.

(ii) If $\alpha=n-1, n \in \mathbb{N}$, they become the usual definitions

$$
{ }_{a} D_{t}^{n-1} f(t)=f^{n-1}(t), \quad{ }_{t} D_{b}^{n-1} f(t)=(-1)^{n} f^{n-1}(t), \quad t \in[a, b] .
$$

Definition $2.3([2,3])$ Let $\alpha \geq 0$ and $n \in \mathbb{N}$.

(i) If $\alpha \in(n-1, n)$ and $f \in A C^{n}\left([a, b], \mathbb{R}^{N}\right)$, then the left and right Caputo fractional derivatives of order $\alpha$ for a function $f$ denoted by ${ }_{a}^{c} D_{t}^{\alpha} f(t)$ and ${ }_{t}^{c} D_{b}^{\alpha} f(t)$, respectively, exist almost everywhere on $[a, b],{ }_{a}^{c} D_{t}^{\alpha} f(t)$ and ${ }_{t}^{c} D_{b}^{\alpha} f(t)$ are represented by

$$
{ }_{a}^{c} D_{t}^{\alpha} f(t)={ }_{a} I_{t}^{n-\alpha} \frac{d^{n}}{d t^{n}} f(t), \quad{ }_{t}^{c} D_{b}^{\alpha} f(t)=(-1)^{n}{ }_{t} I_{b}^{n-\alpha} \frac{d^{n}}{d t^{n}} f(t), \quad t \in[a, b] .
$$

(ii) If $\alpha=n-1$ and $f \in A C^{n-1}\left([a, b], \mathbb{R}^{N}\right)$, then ${ }_{a}^{c} D_{t}^{n-1} f(t)$ and ${ }_{t}^{c} D_{b}^{n-1} f(t)$ are represented by

$$
{ }_{a}^{c} D_{t}^{n-1} f(t)=f^{(n-1)}(t), \quad{ }_{t}^{c} D_{b}^{n-1} f(t)=(-1)^{(n-1)} f^{(n-1)}(t), \quad t \in[a, b] .
$$


Proposition 2.4 ([2, 3]) Let $n \in \mathbb{N}$ and $n-1<\alpha<n$. Iff is a function defined on $[a, b]$, for which the Caputo fractional derivatives ${ }_{a}^{c} D_{t}^{\alpha} f(t)$ and ${ }_{t}^{c} D_{b}^{\alpha} f(t)$ of order $\alpha$ exist together with the Riemann-Liouville fractional derivatives ${ }_{a} D_{t}^{\alpha} f(t)$ and ${ }_{t} D_{b}^{\alpha} f(t)$, then

$$
\begin{aligned}
& { }_{a}^{c} D_{t}^{\alpha} f(t)={ }_{a} D_{t}^{\alpha} f(t)-\sum_{j=0}^{n-1} \frac{f^{j}(a)}{\Gamma(j-\alpha+1)}(t-a)^{j-\alpha}, \quad t \in[a, b], \\
& { }_{t}^{c} D_{b}^{\alpha} f(t)={ }_{t} D_{b}^{\alpha} f(t)-\sum_{j=0}^{n-1} \frac{f^{j}(b)}{\Gamma(j-\alpha+1)}(b-t)^{j-\alpha}, \quad t \in[a, b] .
\end{aligned}
$$

Remark 2.5 In view of (2.1) and (2.2), it is easy to find that ${ }_{0}^{c} D_{t}^{\alpha} u(t)={ }_{0} D_{t}^{\alpha} u(t),{ }_{t}^{c} D_{T}^{\alpha} u(t)=$ ${ }_{t} D_{T}^{\alpha} u(t), t \in[0, T]$ by $u(0)=u(T)=0$.

Proposition 2.6 ([3]) We have the following property of fractional integration:

$$
\int_{a}^{b}\left[{ }_{a} I_{t}^{\alpha} f(t)\right] g(t) d t=\int_{a}^{b}\left[{ }_{t} I_{b}^{\alpha} g(t)\right] f(t) d t, \quad \alpha>0,
$$

provided that $f \in L^{p}\left([a, b], \mathbb{R}^{N}\right), g \in L^{q}\left([a, b], \mathbb{R}^{N}\right)$ and $p \geq 1, q \geq 1,1 / p+1 / q \leq 1+\alpha$ or $p \neq 1, q \neq 1,1 / p+1 / q=1+\alpha$.

Definition 2.7 ([17]) Let $0<\alpha \leq 1$ and $u, v \in L^{1}[0, T]$. For any $\varphi \in C_{0}^{\infty}([0, T], \mathbb{R})$, we have

$$
\int_{0}^{T} v \varphi d t=\int_{0}^{T} u_{t} D_{T}^{\alpha} \varphi d t
$$

so $v$ is said to be an $\alpha$-weak fractional derivative for $u$, and there exists a left RiemannLiouville derivative such that $v={ }_{0} D_{t}^{\alpha} u$.

Define the following fractional-type Sobolev space:

$$
E^{\alpha, p}=\left\{u \in L^{p}([0, T], \mathbb{R}):{ }_{0} D_{t}^{\alpha} u \in L^{p}([0, T], \mathbb{R})\right\}
$$

with the norm

$$
\|u\|_{\alpha, p}=\left(\int_{0}^{T}\left|{ }_{0} D_{t}^{\alpha} u(t)\right|^{p} d t+\int_{0}^{T}|u(t)|^{p} d t\right)^{1 / p} .
$$

Based on [17], if $1<p<\infty$, it is a reflexive and separable Banach space. Moreover, $E_{0}^{\alpha, p}$ represents the closure of $C_{0}^{\infty}([0, T], \mathbb{R})$ in the norm of $E^{\alpha, p}$. So, $E_{0}^{\alpha, p} \subset E^{\alpha, p}$ is also a reflexive and separable Banach space. Clearly, $E_{0}^{\alpha, p}$ becomes the well-known space $W_{0}^{1, p}$ when $\alpha=1$.

Lemma 2.8 ([13]) Let $0<\alpha \leq 1$ and $1<p<\infty$. For all $u \in E_{0}^{\alpha, p}$, we have

$$
\|u\|_{L^{p}} \leq \frac{T^{\alpha}}{\Gamma(\alpha+1)}\left\|_{0} D_{t}^{\alpha} u\right\|_{L^{p}}
$$

Moreover, if $\alpha>\frac{1}{p}$ and $\frac{1}{p}+\frac{1}{q}=1$, then

$$
\|u\|_{\infty} \leq \frac{T^{\alpha-1 / q}}{\Gamma(\alpha)(q(\alpha-1)+1)^{1 / q}}\left\|_{0} D_{t}^{\alpha} u\right\|_{L^{p}} .
$$


Based on (2.4), we can consider $E_{0}^{\alpha, p}$ with respect to the norm

$$
\|u\|_{\alpha, p}=\left(\int_{0}^{T}\left|{ }_{0} D_{t}^{\alpha} u(t)\right|^{p} d t\right)^{1 / p}=\left\|_{0} D_{t}^{\alpha} u\right\|_{L^{p}}, \quad \forall u \in E_{0}^{\alpha, p}
$$

and (2.3) is equivalent to (2.6).

For our problem, we define a new norm in $E_{0}^{\alpha, p}$ by

$$
\|u\|=\left(\int_{0}^{T}\left|{ }_{0} D_{t}^{\alpha} u(t)\right|^{p} d t+\int_{0}^{T} a(t)|u(t)|^{p} d t\right)^{1 / p}
$$

Since ess $\inf _{t \in[0, T]} a(t)>-\lambda_{1}$, following [28], the norm $\|u\|$ is equivalent to $\|u\|_{\alpha, p}$.

Proposition 2.9 ([13]) Let $0<\alpha \leq 1$ and $1<p<\infty$. Assume that $\alpha>\frac{1}{p}$ and the sequence $u_{n}$ converges weakly to $u$ in $E_{0}^{\alpha, p}$, i.e., $u_{n} \rightarrow u$. Then $u_{n} \rightarrow u$ in $C([0, T], \mathbb{R})$, i.e., $\left\|u_{n}-u\right\|_{\infty} \rightarrow 0$, $n \rightarrow \infty$.

Based on the above proposition, there exists a constant $S>0$ such that $\|u\|_{\infty} \leq S\|u\|$. For $v \in E_{0}^{\alpha, p}$, multiplying the two sides of equation (1.1) by $v$ and integrating from 0 to $T$, one has

$$
\begin{aligned}
& \int_{0}^{T}{ }_{t} D_{T}^{\alpha} \phi_{p}\left({ }_{0} D_{t}^{\alpha} u(t)\right) v(t)+\int_{0}^{T} a(t) \phi_{p}(u(t)) u(t) v(t) d t \\
& -\int_{0}^{T} f(t, u(t)) v(t) d t-\mu \int_{0}^{T} b(t)|u(t)|^{\nu-2} u(t) v(t) d t=0 .
\end{aligned}
$$

Based on Proposition 2.6, we have

$$
\begin{aligned}
\int_{0}^{T}{ }_{t} D_{T}^{\alpha} \phi_{p}\left({ }_{0} D_{t}^{\alpha} u(t)\right) v(t) d t & \\
= & -\sum_{j=0}^{m} \int_{t_{j}}^{t_{j+1}} v(t) d\left[{ }_{t} I_{T}^{1-\alpha} \phi_{p}\left({ }_{0} D_{t}^{\alpha} u(t)\right)\right] \\
= & -\left.\sum_{j=0}^{m}{ }_{t} I_{T}^{1-\alpha} \phi_{p}\left({ }_{0} D_{t}^{\alpha} u(t)\right) v(t)\right|_{t_{j}} ^{t_{j+1}}+\sum_{j=0}^{m} \int_{t_{j}}^{t_{j+1}} \phi_{p}\left({ }_{0} D_{t}^{\alpha} u(t)\right)_{0} D_{t}^{\alpha} v(t) d t \\
= & \sum_{j=1}^{m}\left({ }_{t} I_{T}^{1-\alpha} \phi_{p}\left({ }_{0} D_{t}^{\alpha} u\left(t_{j}^{+}\right)\right) v\left(t_{j}\right)-{ }_{t} I_{T}^{1-\alpha} \phi_{p}\left({ }_{0} D_{t}^{\alpha} u\left(t_{j}^{-}\right)\right) v\left(t_{j}\right)\right) \\
& +\int_{0}^{T} \phi_{p}\left({ }_{0} D_{t}^{\alpha} u(t)\right)_{0} D_{t}^{\alpha} v(t) d t \\
= & I_{j}\left(u\left(t_{j}\right)\right) v\left(t_{j}\right)+\int_{0}^{T} \phi_{p}\left({ }_{0} D_{t}^{\alpha} u(t){ }_{0} D_{t}^{\alpha} v(t) d t .\right.
\end{aligned}
$$

Now, we describe the definition of weak solution of (1.1). 
Definition 2.10 Let $u \in E_{0}^{\alpha, p}$ be a weak solution of (1.3) if

$$
\begin{gathered}
\int_{0}^{T} \phi_{p}\left({ }_{0} D_{t}^{\alpha} u(t)\right)_{0} D_{t}^{\alpha} v(t) d t+\int_{0}^{T} a(t) \phi_{p}(u(t)) u(t) v(t) d t+\sum_{j=1}^{m} I_{j}\left(u\left(t_{j}\right)\right) v\left(t_{j}\right) \\
-\int_{0}^{T} f(t, u(t)) v(t) d t-\mu \int_{0}^{T} b(t)|u(t)|^{\nu-2} u(t) v(t) d t=0
\end{gathered}
$$

holds for any $v \in E_{0}^{\alpha, p}$.

Define the functional $\Phi: E_{0}^{\alpha, p} \rightarrow \mathbb{R}$ by

$$
\Phi(u)=\frac{1}{p}\|u\|^{p}+\sum_{j=1}^{m} \int_{0}^{u\left(t_{j}\right)} I_{j}(t) d t-\int_{0}^{T} F(t, u(t)) d t-\frac{\mu}{v} \int_{0}^{T} h(t)|u(t)|^{v} d t
$$

Based on the continuity of $f$ and $I_{j}$, following [31], one has $\phi \in C^{1}\left(E_{0}^{\alpha, p}, \mathbb{R}\right)$. For any $v \in E_{0}^{\alpha, p}$, we can get

$$
\begin{aligned}
\left\langle\Phi^{\prime}(u), v\right\rangle= & \int_{0}^{T}{ }_{t} D_{T}^{\alpha} \phi_{p}\left({ }_{0} D_{t}^{\alpha} u(t)\right) v(t)+\int_{0}^{T} a(t) \phi_{p}(u(t)) u(t) v(t) d t+\sum_{j=1}^{m} I_{j}\left(u\left(t_{j}\right)\right) v\left(t_{j}\right) \\
& -\int_{0}^{T} f(t, u(t)) v(t) d t-\mu \int_{0}^{T} b(t)|u(t)|^{v-2} u(t) v(t) d t .
\end{aligned}
$$

Therefore, the weak solutions of problem (1.1) are the corresponding critical points of $\Phi$. In order to obtain our main results, we introduce the following tools.

Definition 2.11 ([32]) Let $X$ be a Banach space and $\Phi \in C^{1}(X, \mathbb{R})$ satisfy $\Phi(-u)=\Phi(u)$, $u \in X$. Let

$$
\Sigma=\{A \subset X-\{0\} \mid A \text { is closed in } X \text { and symmetric with respect to } 0\}
$$

The genus of $A$ is defined by

$$
\gamma(A)=\left\{\begin{array}{l}
\inf \left\{n \in Z_{+} \mid \text {there exists an odd mapping } \varphi \in C\left(A, \mathbb{R}^{n} \backslash\{0\}\right)\right\} \\
0, \quad A=\emptyset \\
+\infty, \quad \text { when } \varphi \text { is non-existent. }
\end{array}\right.
$$

Lemma $2.12([32])$ Let $n, k \in \mathbb{N}$. The properties of the genus $\gamma$ are as follows:

(i) If $X=X_{1} \oplus X_{2}, \operatorname{dim} X_{1}=k, \gamma(A)>k$, then $A \cap X_{2} \neq \emptyset$;

(ii) If $\Omega$ is a symmetric bounded domain near zero in $\mathbb{R}^{n}$, there exists a mapping $h \in C(A, \partial \Omega)$ with odd homeomorphism such that, for $A \in \Sigma$, then $\gamma(A)=n$;

(iii) If $\gamma(A)=k, 0 \notin A$, then there exist at least $k$ pairs of different points in $A$.

Lemma 2.13 ([32]) Let $X$ be a Banach space and $\Phi \in C^{1}(X, \mathbb{R})$ satisfy $\Phi(-u)=\Phi(u), u \in X$. The pseudo-index is defined by

$$
i^{*}(A)=\inf _{h \in \Lambda_{*}(\rho)} \gamma\left(A \cap h\left(\partial B_{1}\right)\right)
$$


where

$$
\begin{aligned}
& \Lambda_{*}(\rho) \equiv\{h \in C(X, X) \mid h \text { is an odd homeomorphism, } \\
&\text { for some } \left.\rho>0, h\left(B_{1}\right) \subset \Phi^{-1}(0, \infty) \cup B_{\rho}\right\}, \\
& A \in \Sigma^{*} \equiv\{A \in \Sigma \mid A \text { is compact }\} .
\end{aligned}
$$

Suppose that $\Phi$ satisfies the $(P S)_{c}$-condition and

(i) there are constants $\rho, \sigma>0$ such that $\left.\Phi\right|_{X_{1}^{\perp} \cap \partial B_{\rho}} \geq \sigma$ for the subspace $X_{1} \subset X$, $\operatorname{dim} X_{1}=m_{1}$;

(ii) there is a subspace $X_{2} \subset X, \operatorname{dim} X_{2}=m_{2}>m_{1}, l>0$ such that $\Phi(u) \leq 0, \forall u \in X_{2} \backslash B_{l}$.

Then $\Phi$ at least possesses $m_{2}-m_{1}$ pairs of different critical points:

$$
c_{n}^{*}=\inf _{i^{*}(A) \geq n} \sup _{u \in A} \Phi(u) .
$$

Definition 2.14 ([32,33]) Let $X$ be a real Banach space with its dual $X^{*}$ and $\Phi \in C^{1}(X, \mathbb{R})$. For any $\left\{u_{n}\right\} \subset X,\left\{u_{n}\right\}$ has a convergent subsequence if $\Phi\left(u_{n}\right)$ is bounded or $\Phi\left(u_{n}\right) \rightarrow c, c \in$ $\mathbb{R}$ and $\Phi^{\prime}\left(u_{n}\right) \rightarrow 0$ as $n \rightarrow \infty$. Then we say that $\Phi(u)$ satisfies the Palais-Smale condition or the Palais-Smale condition at the level $c\left((\mathrm{PS})\right.$-condition or (PS) ${ }_{c}$-condition for short).

Lemma 2.15 ([34]) Let $E$ be a real Banach space and $\Phi \in C^{1}(E, \mathbb{R})$ satisfy the (PS)condition. If $\Phi$ is bounded from below, then $c=\inf _{E} \Phi$ is a critical point.

\section{Main results}

In order to prove the theorem, we need the following lemmas. Firstly, in $E_{0}^{\alpha, p}$, we can choose a completely orthonormal basis $\left\{e_{i}\right\}_{i=1}^{\infty}$. Set $Y_{i}=\mathbb{R} e_{i}, X_{k}=\bigoplus_{i=1}^{k} Y_{i}, X_{k+r}=\bigoplus_{i=1}^{k+r} Y_{i}$ and $X_{k}^{\perp}=\overline{\bigoplus_{i=k+1}^{\infty} Y_{i}}$, so $W_{T}^{1,2}=X_{k}^{\perp} \oplus X_{k}$ and $\operatorname{dim} X_{k+r}-\operatorname{dim} X_{k}=r$, where $r \in \mathbb{N}$. $b^{ \pm}(t)=$ $\max \{ \pm b(t), 0\}$.

Lemma 3.1 If the assumptions of Theorem 1.2 hold, then $\Phi$ satisfies the $(P S)_{c}$-condition for $\mu \in(0, \infty)$.

Proof Let $\left\{u_{n}\right\}_{n \in \mathbb{N}} \subset E_{0}^{\alpha, p}$ such that $\Phi\left(u_{n}\right) \rightarrow c$ and $\Phi^{\prime}\left(u_{n}\right) \rightarrow 0$ as $n \rightarrow \infty$, which tell us the fact that there exists a constant $C>0$ such that $\left|\Phi\left(u_{n}\right)\right| \leq C,\left\|\Phi^{\prime}\left(u_{n}\right)\right\|_{\left(E_{0}^{\alpha, p}\right)^{*}} \leq C$. Next, our aim is to prove that $\left\{u_{n}\right\}$ is a bounded sequence in $E_{0}^{\alpha, p}$. If not, we assume that $\left\|u_{n}\right\| \rightarrow \infty$ as $n \rightarrow \infty$. For any $u \in E_{0}^{\alpha, p} \backslash\{0\}$, let $v_{n}=\frac{u_{n}}{\left\|u_{n}\right\|}$, then $v_{n}$ is bounded in $E_{0}^{\alpha, p}$. From the fact that $E_{0}^{\alpha, p}$ is a reflexive Banach space, we can find a subsequence of $\left\{v_{n}\right\}$ (called again $\left\{v_{n}\right\}$ ) such that $v_{n} \rightarrow v$ in $E_{0}^{\alpha, p}, v_{n} \rightarrow v$ uniformly in $C([0, T], \mathbb{R})$. By $\left(\mathrm{H}^{\prime}\right)$, we can get

$$
\begin{aligned}
\int_{0}^{T} F\left(t, u_{n}\right) d t= & \frac{1}{p}\left\|u_{n}\right\|^{p}+\sum_{j=1}^{m} \int_{0}^{u_{n}\left(t_{j}\right)} I_{j}(t) d t-\frac{\mu}{v} \int_{0}^{T} b(t)|u(t)|^{v} d t-\Phi\left(u_{n}\right) \\
\leq & \frac{1}{p}\left\|u_{n}\right\|^{p}+\sum_{j=1}^{m} a_{j} S\left\|u_{n}\right\|+\sum_{j=1}^{m} d_{j} S^{\gamma_{j}+1}\left\|u_{n}\right\|^{\gamma_{j}+1} \\
& +\frac{\mu}{v} T S^{v}\|b\|_{\infty}\|u\|^{v}+C,
\end{aligned}
$$


which shows the fact that there exists $c>0$ such that

$$
\left\{\begin{array}{l}
\lim _{n \rightarrow \infty} \int_{0}^{T} \frac{F\left(t, u_{n}\right)}{\left\|u_{n}\right\|^{p}} d t \leq c \quad \text { for } \gamma_{j} \in[0, p-1) \\
\lim _{n \rightarrow \infty} \int_{0}^{T} \frac{F\left(t, u_{n}\right)}{\left\|u_{n}\right\|^{\gamma *+1}} d t \leq c \quad \text { for } \gamma_{j} \in[p-1, \theta-1)
\end{array}\right.
$$

where $\gamma_{*}=\max \left\{\gamma_{j}\right\}$. From (H1), for any $\varepsilon>0$, there exists a constant $D_{1}>0$ such that

$$
|f(t, u)| \leq \varepsilon|u|^{p-1}, \quad|u| \leq D_{1} .
$$

Therefore, for $|u| \leq D_{1}$, there exists a constant $\varepsilon_{1}>0$ such that

$$
|u f(t, u)-\theta F(t, u)| \leq \varepsilon_{1}(1+\theta) u^{p} .
$$

For $(t, u) \in[0, T] \times\left[D_{1}, D\right]$, we can find a constant $c>0$ such that

$$
|u f(t, u)-\theta F(t, u)| \leq c,
$$

which together with $\left(\mathrm{H}^{\prime}\right)$ yields that

$$
u f(t, u)-\theta F(t, u) \geq-\varepsilon_{1}(1+\theta) u^{p}-c .
$$

Based on (H4), there exists a constant $c>0$ such that

$$
\theta \sum_{j=1}^{m} \int_{0}^{u_{n}\left(t_{j}\right)} I_{j}(t) d t-\sum_{j=1}^{m} I_{j}\left(u_{n}\left(t_{j}\right)\right) u_{n}\left(t_{j}\right) \geq-c .
$$

Therefore, we have

$$
\begin{aligned}
\theta C+C\left\|u_{n}\right\| \geq & \theta \Phi\left(u_{n}\right)-\left\langle\Phi^{\prime}\left(u_{n}\right), u_{n}\right\rangle \\
= & \left(\frac{\theta}{p}-1\right)\left\|u_{n}\right\|^{p}+\theta \sum_{j=1}^{m} \int_{0}^{u_{n}\left(t_{j}\right)} I_{j}(t) d t-\sum_{j=1}^{m} I_{j}\left(u_{n}\left(t_{j}\right)\right) u_{n}\left(t_{j}\right) \\
& +\int_{0}^{T}\left(u_{n}(t) f\left(t, u_{n}(t)\right)-\theta F\left(t, u_{n}(t)\right)\right) d t \\
& -\mu \frac{\theta-v}{v} \int_{0}^{T} b(t)\left|u_{n}(t)\right|^{v} d t \\
\geq & \left\|u_{n}\right\|^{p}+\int_{0}^{T}\left(u_{n}(t) f\left(t, u_{n}(t)\right)-\theta F\left(t, u_{n}(t)\right)\right) d t \\
& -\mu \frac{\theta-v}{v} \int_{0}^{T} b(t)\left|u_{n}(t)\right|^{v} d t-c \\
\geq & \left\|u_{n}\right\|^{p}-\varepsilon_{1}(1+\theta) T\left\|u_{n}\right\|_{\infty}^{p}-\mu \frac{\theta-v}{v}\left\|b^{+}\right\|_{L^{1}}\left\|u_{n}\right\|_{\infty}^{v}-c .
\end{aligned}
$$

This shows the fact that there exists a constant $c>0$ such that

$$
\lim _{n \rightarrow \infty}\left\|v_{n}\right\|_{\infty}=\lim _{n \rightarrow \infty} \frac{\left\|u_{n}\right\|_{\infty}}{\left\|u_{n}\right\|} \geq c>0 .
$$


Hence, we obtain $v \neq 0$. From $\left(\mathrm{H}^{\prime}\right)$, for $s \in[D /|u|, 1],|u| \geq D$, one has

$$
\frac{d}{d s}\left(\frac{F(t, s u)}{s^{\theta}}\right)=\frac{f(t, s u) s u-\theta F(t, s u)}{s^{\theta+1}} \geq 0 .
$$

Thus, for $t \in[0, T]$, we have

$$
F(t, u) \geq \frac{|u|^{\theta}}{D^{\theta}} \inf _{|u|=D} F(t, u)
$$

which together with $\left(\mathrm{H} 2^{\prime}\right)$ yields that

$$
\left\{\begin{array}{l}
\frac{F(t, u)}{|u|^{p}} \geq \frac{|u|^{\theta-p}}{D^{\theta}} \inf _{|u|=D} F(t, u) \rightarrow \infty \quad \text { as }|u| \rightarrow \infty \text { for } \gamma_{j} \in[0, p-1) \\
\frac{F(t, u)}{|u|^{\gamma_{*}+1}} \geq \frac{|u|^{\theta-\gamma_{*}-1}}{D^{\theta}} \inf _{|u|=D} F(t, u) \rightarrow \infty \quad \text { as }|u| \rightarrow \infty \text { for } \gamma_{j} \in[p-1, \theta-1)
\end{array}\right.
$$

Based on Fatou's lemma, one has

$$
\left\{\begin{array}{l}
\int_{v \neq 0} \frac{F\left(t, u_{n}\right)}{\left|u_{n}\right|^{p}}\left|v_{n}\right|^{p} d t \rightarrow \infty \quad \text { as } n \rightarrow \infty \text { for } \gamma_{j} \in[0, p-1), \\
\int_{v \neq 0} \frac{F\left(t, u_{n}\right)}{\left|u_{n}\right|^{\gamma *+1}}\left|v_{n}\right|^{\gamma_{*}+1} d t \rightarrow \infty \quad \text { as } n \rightarrow \infty \text { for } \gamma_{j} \in[p-1, \theta-1) .
\end{array}\right.
$$

In view of (3.3), we know $F(t, u) \geq 0$ for $|u| \geq D, t \in[0, T]$. From (H1), there exist constants $c, \varepsilon_{2}>0$ such that

$$
F(t, u) \geq-\varepsilon_{2} u^{p}-c, \quad u \in \mathbb{R}, t \in[0, T]
$$

Let

$$
\mathcal{O}:=\operatorname{meas}\{t \in[0, T]: v(t)=0\}
$$

We have

$$
\left\{\begin{array}{l}
\int_{v=0} \frac{F\left(t, u_{n}\right)}{\left\|u_{n}\right\|^{p}} d t \geq-\varepsilon_{2} S^{p} \mathcal{O}-\frac{c}{\left\|u_{n}\right\|^{p}} \quad \text { for } \gamma_{j} \in[0, p-1) \\
\int_{v=0} \frac{F\left(t, u_{n}\right)}{\left\|u_{n}\right\|^{\gamma_{*}+1}} d t \geq \frac{-\varepsilon_{2} S^{p} \mathcal{O}}{\left\|u_{n}\right\|^{\gamma_{*}+1-p}}-\frac{c}{\left\|u_{n}\right\|^{\gamma_{*}+1}} \quad \text { for } \gamma_{j} \in[p-1, \theta-1)
\end{array}\right.
$$

This means

$$
\begin{cases}\liminf _{n \rightarrow \infty} \int_{v=0} \frac{F\left(t, u_{n}\right)}{\left\|u_{n}\right\|^{p}} d t>-\infty & \text { for } \gamma_{j} \in[0, p-1) \\ \liminf _{n \rightarrow \infty} \int_{v=0} \frac{F\left(t, u_{n}\right)}{\left\|u_{n}\right\|^{\gamma *+1}} d t>-\infty & \text { for } \gamma_{j} \in[p-1, \theta-1)\end{cases}
$$

Thus, we can obtain

$$
\left\{\begin{aligned}
\liminf _{n \rightarrow \infty} \int_{0}^{T} \frac{F\left(t, u_{n}\right)}{\left\|u_{n}\right\|^{p}} d t & =\liminf _{n \rightarrow \infty}\left(\int_{v=0}+\int_{v \neq 0}\right) \frac{F\left(t, u_{n}\right)}{\left\|u_{n}\right\|^{p}} d t \\
& \rightarrow \infty \text { for } \gamma_{j} \in[0, p-1), \\
\liminf _{n \rightarrow \infty} \int_{0}^{T} \frac{F\left(t, u_{n}\right)}{\left\|u_{n}\right\|^{\gamma_{j}+1}} d t & =\liminf _{n \rightarrow \infty}\left(\int_{v=0}+\int_{v \neq 0}\right) \frac{F\left(t, u_{n}\right)}{\left\|u_{n}\right\|^{\gamma_{j}+1}} d t \\
& \rightarrow \infty \text { for } \gamma_{j} \in[p-1, \theta-1) .
\end{aligned}\right.
$$


This is a contradiction to (3.1). Hence, $\left\{u_{n}\right\}$ is bounded. By a similar standard argument as the one in [15], we can show $u_{n} \rightarrow u$ in $E_{0}^{\alpha, p}$. For the completeness, we state the proof as follows. Based on the fact that $E_{0}^{\alpha, p}$ is a reflexive Banach space, $\left\{u_{n}\right\}$ has a convergent subsequence (named again $\left\{u_{n}\right\}$ ) such that $u_{n} \rightarrow u$ in $E_{0}^{\alpha, p}$, so $u_{n} \rightarrow u$ uniformly in $C([0, T], \mathbb{R})$. Thus, we have

$$
\begin{aligned}
& \int_{0}^{T}\left(f\left(t, u_{n}(t)\right)-f(t, u(t))\right)\left(u_{n}(t)-u(t)\right) d t \rightarrow 0, \quad n \rightarrow \infty, \\
& \sum_{j=1}^{m}\left(I_{j}\left(u_{n}\left(t_{j}\right)\right)-I_{j}\left(u\left(t_{j}\right)\right)\right)\left(u_{n}\left(t_{j}\right)-u\left(t_{j}\right)\right) \rightarrow 0, \quad n \rightarrow \infty, \\
& \int_{0}^{T} a(t)\left(\phi_{p}\left(u_{n}(t)\right)-\phi_{p}(u(t))\right)\left(u_{n}(t)-u(t)\right) d t \rightarrow 0, \quad n \rightarrow \infty, \\
& \int_{0}^{T} b(t)\left(\left|u_{n}\right|^{\nu-2} u_{n}(t)-\left|u_{n}(t)\right|^{\nu-2} u_{n}(t)\right)\left(u_{n}(t)-u(t)\right) d t \rightarrow 0, \quad n \rightarrow \infty .
\end{aligned}
$$

By $\Phi^{\prime}\left(u_{n}\right) \rightarrow 0$ and $u_{n} \rightarrow u$, we can obtain

$$
\left\langle\Phi^{\prime}\left(u_{n}\right)-\Phi^{\prime}(u), u_{n}-u\right\rangle \rightarrow 0, \quad n \rightarrow \infty
$$

Thus, we have

$$
\begin{aligned}
& \left\langle\Phi^{\prime}\left(u_{n}\right)-\Phi^{\prime}(u), u_{n}-u\right\rangle \\
& =\int_{0}^{T}\left(\phi_{p}\left({ }_{0} D_{t}^{\alpha} u_{n}(t)\right)-\phi_{p}\left({ }_{0} D_{t}^{\alpha} u(t)\right)\right)\left({ }_{0} D_{t}^{\alpha} u_{n}(t)-{ }_{0} D_{t}^{\alpha} u(t)\right) d t \\
& \quad+\int_{0}^{T} a(t)\left(\phi_{p}\left(u_{n}(t)\right)-\phi_{p}(u(t))\right)\left(u_{n}(t)-u(t)\right) d t \\
& \quad+\sum_{j=1}^{m}\left(I_{j}\left(u_{n}\left(t_{j}\right)\right)-I_{j}\left(u\left(t_{j}\right)\right)\right)\left(u_{n}\left(t_{j}\right)-u\left(t_{j}\right)\right) \\
& \quad-\int_{0}^{T}\left(f\left(t, u_{n}(t)\right)-f(t, u(t))\right)\left(u_{n}(t)-u(t)\right) d t \\
& \quad-\mu \int_{0}^{T} b(t)\left(\left|u_{n}(t)\right|^{\nu-2} u_{n}(t)-\left|u_{n}(t)\right|^{\nu-2} u_{n}(t)\right)\left(u_{n}(t)-u(t)\right) d t .
\end{aligned}
$$

Based on [35], we have

$$
\begin{gathered}
\int_{0}^{T}\left(\phi_{p}\left({ }_{0} D_{t}^{\alpha} u_{n}(t)\right)-\phi_{p}\left({ }_{0} D_{t}^{\alpha} u(t)\right)\right)\left({ }_{0} D_{t}^{\alpha} u_{n}(t)-{ }_{0} D_{t}^{\alpha} u(t)\right) d t \\
\geq \begin{cases}\left.c \int_{0}^{T}\right|_{0} D_{t}^{\alpha} u_{n}(t)-\left.{ }_{0} D_{t}^{\alpha} u(t)\right|^{p} d t, & p \geq 2, \\
c \int_{0}^{T} \frac{{ }_{0} D_{t}^{\alpha} u_{n}(t)-\left.0 D_{t}^{\alpha} u(t)\right|^{2}}{\left({ }_{0} D_{t}^{\alpha} u_{n}(t)|+|_{0} D_{t}^{\alpha} u(t)\right)^{2-p}} d t, & 1<p<2 .\end{cases}
\end{gathered}
$$


If $p \geq 2$, following (3.5)-(3.10), $\left\|u_{n}-u\right\| \rightarrow 0, n \rightarrow \infty$. If $1<p<2$, by Hölder's inequality, one has

$$
\begin{aligned}
& \int_{0}^{T}\left|{ }_{0} D_{t}^{\alpha} u_{n}(t)-{ }_{0} D_{t}^{\alpha} u(t)\right|^{p} d t \\
& \quad \leq c\left(\int_{0}^{T} \frac{\left|{ }_{0} D_{t}^{\alpha} u_{n}(t)-{ }_{0} D_{t}^{\alpha} u(t)\right|^{2}}{\left({ }_{0} D_{t}^{\alpha} u_{n}(t)\left|+{ }_{0} D_{t}^{\alpha} u(t)\right|\right)^{2-p}} d t\right)^{\frac{p}{2}}\left(\left\|u_{n}\right\|+\|u\|\right)^{\frac{p(2-p)}{2}} .
\end{aligned}
$$

Thus, we can get

$$
\begin{gathered}
\int_{0}^{T}\left(\phi_{p}\left({ }_{0} D_{t}^{\alpha} u_{n}(t)\right)-\phi_{p}\left({ }_{0} D_{t}^{\alpha} u(t)\right)\right)\left({ }_{0} D_{t}^{\alpha} u_{n}(t)-{ }_{0} D_{t}^{\alpha} u(t)\right) d t \\
\geq \frac{c}{\left(\left\|u_{n}\right\|+\|u\|\right)^{2-p}}\left(\int_{0}^{T}\left|{ }_{0} D_{t}^{\alpha} u_{n}(t)-{ }_{0} D_{t}^{\alpha} u(t)\right|^{p} d t\right)^{\frac{2}{p}} .
\end{gathered}
$$

Hence, one has $\left\|u_{n}-u\right\| \rightarrow 0, n \rightarrow \infty$. Therefore, $\Phi(u)$ satisfies the (PS) ${ }_{c}$-condition.

Lemma 3.2 If the assumptions of Theorem 1.2 hold, then there exist constants $\rho, \sigma, \mu_{*}>0$ such that $\left.\Phi\right|_{X_{k}^{\perp} \cap \partial B_{\rho}} \geq \sigma$ for $\mu \in\left[0, \mu_{*}\right)$.

Proof By (H1) and (H7), for any $\varepsilon>0$, there exists $C_{\varepsilon}$ such that, for $x \in \mathbb{R}, t \in[0, T]$,

$$
F(t, u) \leq \varepsilon|u|^{p}+C_{\varepsilon}|u|^{\beta}
$$

which implies that

$$
\begin{aligned}
\int_{0}^{T} F(t, u) d t & \leq \varepsilon \int_{0}^{T}|u|^{p} d t+C_{\varepsilon} \int_{0}^{T}|u|^{\beta} d t \\
& \leq \varepsilon T S^{p}\|u\|^{p}+C_{\varepsilon} T S^{\beta}\|u\|^{\beta} .
\end{aligned}
$$

Thus, from (H5), we can obtain

$$
\begin{aligned}
\Phi(u) & =\frac{1}{p}\|u\|^{p}+\sum_{j=1}^{m} \int_{0}^{u\left(t_{j}\right)} I_{j}(t) d t-\int_{0}^{T} F(t, u(t)) d t-\frac{\mu}{v} \int_{0}^{T} b(t)|u(t)|^{v} d t \\
& \geq \frac{1}{p}\|u\|^{p}-\int_{0}^{T} F(t, u(t))-\frac{\mu}{v} \int_{0}^{T} b^{+}(t)|u(t)|^{v} d t \\
& \geq \frac{1}{p}\|u\|^{p}-\varepsilon T S^{p}\|u\|^{p}-C_{\varepsilon} T S^{\beta}\|u\|^{\beta}-\frac{\mu}{v} T S^{v}\left\|b^{+}\right\|_{L^{1}}\|u\|^{v} \\
& =\|u\|^{v}\left(\left(\frac{1}{p}-\varepsilon T S^{p}\right)\|u\|^{p-v}-C_{\varepsilon} T S^{\beta}\|u\|^{\beta-v}-\frac{\mu}{v} T S^{v}\left\|b^{+}\right\|_{L^{1}}\right) .
\end{aligned}
$$

Choosing $\varepsilon=\frac{5}{6 T_{p} S^{p}}$, we have

$$
\varphi(u) \geq\|u\|^{\nu}\left(\frac{1}{6 p}\|u\|^{p-\nu}-C_{\varepsilon} T S^{\beta}\|u\|^{\beta-\nu}-\frac{\mu}{v} T S^{\nu}\left\|b^{+}\right\|_{L^{1}}\right) .
$$


Let

$$
y(t)=\frac{1}{6 p} t^{p-\nu}-C_{\varepsilon} T S^{\beta} t^{\beta-\nu}, \quad t \geq 0 .
$$

By a simple calculation, we can find

$$
\rho=\left[\frac{p-v}{6 p C_{\varepsilon} T S^{\beta}(\beta-v)}\right]^{\frac{1}{\beta-p}}
$$

such that

$$
y(\rho)=\max _{t \geq 0} y(t)=\frac{\beta-p}{6 p(\beta-v)}\left[\frac{p-v}{6 p C_{\varepsilon} T S^{\beta}(\beta-v)}\right]^{\frac{p-v}{\beta-p}}>0 .
$$

Thus, there exists a constant

$$
\mu_{*}=\frac{v(\beta-p)}{6 p T S^{v}(\beta-v)\left\|b^{+}\right\|_{L^{1}}}\left[\frac{p-v}{6 p C_{\varepsilon} T S^{\beta}(\beta-v)}\right]^{\frac{p-v}{\beta-p}} .
$$

When $\mu \in\left[0, \mu_{*}\right)$, we can find a constant $\sigma>0$ such that

$$
\left.\Phi\right|_{X_{k}^{\perp} \cap \partial B_{\rho}} \geq \sigma .
$$

Lemma 3.3 If the assumptions of Theorem 1.2 hold, then there exists a constant $l>0$ such that $\Phi(u) \leq 0, \forall u \in X_{k+r} \backslash B_{l}$ for $\mu \in(0, \infty)$.

Proof For $t \in[0, T]$, we know that

$$
F(t, u) \geq \frac{|u|^{\theta}}{D^{\theta}} \inf _{|u|=D} F(t, u) .
$$

Thus, we have

$$
F(t, u) \geq k|u|^{\theta}, \quad|u| \geq D, t \in[0, T]
$$

where $k=D^{-\theta} \inf _{|u|=D} F(t, u)>0$. By $(\mathrm{H} 1)$, there exist constants $\varepsilon_{3}, c>0$ such that

$$
F(t, u) \geq-\varepsilon_{3} u^{p}-c, \quad|u| \leq D, t \in[0, T] .
$$

Hence, one has

$$
F(t, u) \geq k|u|^{\theta}-l u^{p}-c, \quad u \in \mathbb{R}, t \in[0, T],
$$

where $l=k D^{\theta-p}+\varepsilon_{3}$. Thus, for any $u \in X_{k+j}$, by the equivalence of the norms on the finitedimensional space, there exist constants $l_{1}, l_{2}>0$ such that

$$
\begin{aligned}
\varphi(u) \leq & \frac{1}{p}\|u\|^{p}+\sum_{j=1}^{m} a_{j} S\|u\|+\sum_{j=1}^{m} d_{j} S^{\gamma_{j}+1}\|u\|^{\gamma_{j}+1}+l l_{1}^{p}\|u\|^{p} \\
& +\frac{\mu}{v} T S^{v}\|b\|_{\infty}\|u\|^{v}+c T-k l_{2}^{\theta}\|u\|^{\theta},
\end{aligned}
$$


which implies $\Phi(u) \rightarrow-\infty$ as $\|u\| \rightarrow \infty$ because of $\theta>\left\{p, \gamma_{j}+1\right\}$. There exists a constant $l>0$ such that $\Phi(u) \leq 0, \forall u \in X_{k+r} \backslash B_{l}$ for $\mu \in(0, \infty)$.

Next, we give the proof of Theorem 1.2.

Proof of Theorem 1.2 Based on the genus property and the definition of $c_{n}^{*}$, we have, for $r \in \mathbb{N}$

$$
\sigma \leq c_{k+s}^{*}<+\infty, \quad r \geq s \geq 1
$$

For all $A \in \Sigma^{*}$, we know that $i^{*}(A) \geq k+s$. Let $h_{0}=\rho \cdot i d$, then $h_{0} \in \Lambda_{*}(\rho)$ and

$$
\gamma\left(A \cap \partial B_{\rho}\right)=\gamma\left(A \cap h_{0}\left(\partial B_{1}\right)\right) \geq \inf _{h \in \Lambda_{*}(\rho)} \gamma\left(A \cap h\left(\partial B_{1}\right)\right)=i^{*}(A)>k .
$$

From (i) of Lemma 2.12, one has $A \cap \partial B_{\rho} \cap X_{k}^{\perp} \neq \emptyset$. By Lemma 3.2, we have

$$
\sup _{u \in A} \Phi(u) \geq \inf _{u \in \partial B_{\rho} \cap X_{k}^{\perp}} \Phi(u) \geq \sigma .
$$

In view of the arbitrariness of $A \in \Sigma^{*}$, so $c_{k+s}^{*} \geq \sigma$. Based on Lemma 2.13, if $k+s \leq \operatorname{dim} X_{k+r}$, one has $c_{k+s}^{*}<+\infty$. Then we have

$$
\sigma \leq c_{k+1}^{*} \leq c_{k+2}^{*} \leq \cdots \leq c_{k+r}^{*}<+\infty
$$

From Lemmas 3.1, 3.2 and 3.3, we know that (i) and (ii) of Lemma 2.13 and the (PS) $c_{c^{-}}$ condition are satisfied. Moreover, $\Phi(u)=\Phi(-u)$ and $\Phi(0)=0$. Thus, from Lemma 2.13, $\Phi(u)$ possesses at least $r$ pairs of different critical points. Since $r$ is arbitrary and $\lambda_{r} \rightarrow \infty$, $r \rightarrow \infty$, then problem (1.1) has infinitely many nontrivial weak solutions.

Lemma 3.4 If the assumptions of Theorem 1.5 hold, then problem (1.1) has at least one weak solution for $\mu \in(0, \infty)$.

Proof In view of (H9), for any $\xi>0$, we can find a constant $K>0$ such that, for $t \in[0, T]$,

$$
F(t, u) \leq \xi|u|^{p}, \quad|u| \geq K
$$

Since $f$ is continuous, there exists $c>0$ such that

$$
F(t, u) \leq \xi|u|^{p}+c \quad \text { for }(t, u) \in[0, T] \times \mathbb{R} .
$$

From (H3"), we have

$$
\begin{aligned}
\Phi(u) & =\frac{1}{p}\|u\|^{p}+\sum_{j=1}^{m} \int_{0}^{u\left(t_{j}\right)} I_{j}(t) d t-\int_{0}^{T} F(t, u(t)) d t-\frac{\mu}{v} \int_{0}^{T} b(t)|u(t)|^{v} d t \\
& \geq \frac{1}{p}\|u\|^{p}-\sum_{j=1}^{m} a_{j} S\|u\|-\sum_{j=1}^{m} d_{j} S^{\gamma_{j}+1}\|u\|^{\gamma_{j}+1}-\xi S^{p} T\|u\|^{p}-\frac{\mu}{v}\left\|b^{+}\right\|_{L^{1}} S^{v}\|u\|^{v}-c T .
\end{aligned}
$$


Setting $\xi=\frac{1}{2 p T S^{p}}$, one has

$$
\Phi(u) \geq \frac{1}{2 p}\|u\|^{p}-\sum_{j=1}^{m} a_{j} S\|u\|-\sum_{j=1}^{m} d_{j} S^{\gamma_{j}+1}\|u\|^{\gamma_{j}+1}-\frac{\mu}{v}\left\|b^{+}\right\|_{L^{1}} S^{\nu}\|u\|^{\nu}-c T .
$$

In view of $0<\gamma_{j}<p-1$, it is easy to get that $\Phi(u) \rightarrow \infty$ as $\|u\| \rightarrow \infty$. Therefore, $\Phi(u)$ is coercive, which presents the fact that $\Phi(u)$ is bounded from below. Let $\left\{u_{n}\right\} \subset E_{0}^{\alpha, p}$ such that $\Phi\left(u_{n}\right)$ is bounded and $\Phi^{\prime}\left(u_{n}\right) \rightarrow 0$ as $n \rightarrow \infty$. Since $\Phi(u)$ is coercive, so $\left\{u_{n}\right\}$ is bounded. By a similar way as Lemma 3.1, we can get that $\Phi(u)$ satisfies the (PS)-condition. Therefore, problem (1.1) has at least one weak solution for $\mu \in(0, \infty)$.

Next, we show the proof of Theorem 1.5.

Proof of Theorem 1.5 Let $u_{*} \in E_{0}^{\alpha, p}$ be a critical point of $\Phi(u)$ such that $\Phi\left(u_{*}\right)=\inf _{E_{0}^{\alpha, p}} \Phi(u)$. Based on (H8), we can find a function $v \in E_{0}^{\alpha, p}$ such that

$$
\frac{1}{\mu} \int_{0}^{T} F(t, v(t)) d t+\frac{1}{v} \int_{0}^{T} b(t)|v(t)|^{v} d t>0
$$

Thus, we have

$$
\begin{aligned}
\Phi(u)= & \frac{1}{p}\|v\|^{p}+\sum_{j=1}^{m} \int_{0}^{v\left(t_{j}\right)} I_{j}(t) d t-\int_{0}^{T} F(t, v(t)) d t-\frac{\mu}{v} \int_{0}^{T} b(t)|v(t)|^{v} d t \\
\leq & \frac{1}{p}\|v\|^{p}+\sum_{j=1}^{m} a_{j} S\|v\|+\sum_{j=1}^{m} d_{j} S^{\gamma_{j}+1}\|v\|^{\gamma_{j}+1}-\int_{0}^{T} F(t, v(t)) d t \\
& -\frac{\mu}{v} \int_{0}^{T} b(t)|v(t)|^{v} d t .
\end{aligned}
$$

Therefore, there exists

$$
\mu^{*}=\frac{\frac{1}{p}\|v\|^{p}+\sum_{j=1}^{m} a_{j} S\|v\|+\sum_{j=1}^{m} d_{j} S^{\gamma_{j}+1}\|v\|^{\gamma_{j}+1}}{\frac{1}{\mu} \int_{0}^{T} F(t, v(t)) d t+\frac{1}{v} \int_{0}^{T} b(t)|v(t)|^{v} d t}>0
$$

such that $\Phi(v)<0$ if $\mu>\mu^{*}$. Thus, $\Phi\left(u_{*}\right)=c<0$, which implies the fact that $u_{*}$ is a nontrivial weak solution of problem (1.1).

\section{Conclusion}

In this paper, we are devoted to analyzing a class of nonlinear fractional differential models generated by impulsive effects. By variational methods, we can find the range of controlled parameters to ensure the existence of solutions for this type of differential model when nonlinearity $f$ is $p$-suplinear growth or $p$-sublinear growth. It should be pointed out that if $\lambda=0$ and the nonlinearity $f$ is $p$-suplinear growth, our results enrich and extend some previous results. 


\section{Competing interests}

The authors declare that they have no competing interests.

\section{Authors' contributions}

The authors contributed equally in this article. They have all read and approved the final manuscript.

\section{Author details}

'School of Mathematics, China University of Mining and Technology, Xuzhou, 221116, P.R. China. ${ }^{2}$ Department of Personnel, China University of Mining and Technology, Xuzhou, 221116, P.R. China.

\section{Publisher's Note}

Springer Nature remains neutral with regard to jurisdictional claims in published maps and institutional affiliations.

Received: 21 August 2017 Accepted: 14 November 2017 Published online: 21 November 2017

\section{References}

1. Mainardi, F: Fractional diffusive waves in viscoelastic solids. In: Wegner, JL, Norwood, FR (eds.) Nonlinear Waves in Solids, pp. 93-97. ASME/AMR, Fairfield (1995)

2. Podlubny, I: Fractional Differential Equation. Academic Press, San Diego (1999)

3. Kilbas, AA, Srivastava, HM, Trujillo, JJ: Theory and Applications of Fractional Differential Equations. Elsevier Amsterdam (2006)

4. Szymanek, E: The application of fractional order differential calculus for the description of temperature profiles in a granular layer. In: Mitkowski, W, Kacprzyk, J, Baranowski, J (eds.) Advances in the Theory and Applications of Non-integer Order Systems. LNEE, vol. 275, pp. 243-248. Springer, Cham (2013)

5. Leszczynski, J, Blaszczyk, T: Modeling the transition between stable and unstable operation while emptying a silo. Granul. Matter 13, 429-438 (2011)

6. Chen, T, Liu, W: An anti-periodic boundary value problem for the fractional differential equation with a $p$-Laplacian operator. Appl. Math. Lett. 25, 1671-1675 (2012)

7. Shen, T, Liu, W, Shen, X: Existence and uniqueness of solutions for several BVPs of fractional differential equations with p-Laplacian operator. Mediterr. J. Math. 13, 4623-4637 (2016)

8. Shen, T, Liu, W: Existence of solutions for fractional integral boundary value problems with $p(t)$-Laplacian operator. J. Nonlinear Sci. Appl. 9, 5000-5010 (2016)

9. Agarwal, R, O'Regan, D, Stanek, S: Positive solutions for Dirichlet problems of singular nonlinear fractional differential equations. J. Math. Anal. Appl. 371, 57-68 (2010)

10. Jiang, W: The existence of solutions to boundary value problems of fractional differential equations at resonance. Nonlinear Anal. 74, 1987-1994 (2011)

11. Kosmatov, N: A boundary value problem of fractional order at resonance. Electron. J. Differ. Equ. 2010, 135 (2010)

12. Bai, Z, Zhang, Y: Solvability of fractional three-point boundary value problems with nonlinear growth. Appl. Math. Comput. 218, 1719-1725 (2011)

13. Jiao, F, Zhou, Y: Existence results for fractional boundary value problem via critical point theory. Int. J. Bifurc. Chaos 4(22), 1-17 (2012)

14. Torres, C: Mountain pass solution for fractional boundary value problem. J. Fract. Calc. Appl. 1(5), 1-10 (2014)

15. Chen, T, Liu, W: Solvability of fractional boundary value problem with $p$-Laplacian via critical point theory. Bound. Value Probl. 2016, 75 (2016)

16. Bergounioux, M, Leaci, A, Nardi, G: Fractional Sobolev spaces and bounded variation functions. arXiv:1603.05033

17. Idczak, D, Walczak, S: Fractional Sobolev spaces via Riemann-Liouville derivatives. J. Funct. Spaces Appl. 2013, Article ID 128043 (2013)

18. Jin, H, Liu, W: Eigenvalue problem for fractional differential operator containing left and right fractional derivatives. Adv. Differ. Equ. 2016, 246 (2016)

19. Bonanno, G, Rodríguez-López, R, Tersian, S: Existence of solutions to boundary value problem for impulsive fractional differential equations. Fract. Calc. Appl. Anal. 17(3), 717-744 (2014)

20. Zhang, Z, Yuan, R: An application of variational methods to Dirichlet boundary value problem with impulses Nonlinear Anal. 11, 155-162 (2010)

21. Tian, Y, Ge, W: Applications of variational methods to boundary value problem for impulsive differential equations. Proc. Edinb. Math. Soc. 51, 509-527 (2008)

22. Sun, J, Chen, H, Liu, Y: The existence and multiplicity of solutions for an impulsive differential equation with two parameters via a variational method. Nonlinear Anal. 73, 440-449 (2010)

23. Xu, J, Wei, Z, Ding, Y: Existence of weak solutions for $p$-Laplacian problem with impulsive effects. Taiwan. J. Math. 17, 501-515 (2013)

24. Bai, L, Dai, B: Three solutions for a $p$-Laplacian boundary value problem with impulsive effects. Appl. Math. Comput. 217, 9895-9904 (2011)

25. Nyamoradi, N, Rodríguez-López, R: Multiplicity of solutions to fractional Hamiltonian systems with impulsive effects Chaos Solitons Fractals 102, 254-263 (2017)

26. Nyamoradi, N: Multiplicity of nontrivial solutions for boundary value problem for impulsive fractional differential inclusions via nonsmooth critical point theory. Fract. Calc. Appl. Anal. 18, 1470-1491 (2015)

27. Nyamoradi, N: Existence and multiplicity of solutions for impulsive fractional differential equations. Mediterr. J. Math. (2017). doi:10.1007/s00009-016-0806-5

28. Ledesma, C, Nyamoradi, N: Impulsive fractional boundary value problem with $p$-Laplace operator. J. Appl. Math. Comput. (2017). doi:10.1007/s12190-016-1035-6

29. Ye, Y, Tang, C: Infinitely many solutions for fourth-order elliptic equation. J. Math. Anal. Appl. 394, $841-854$ (2012) 
30. Zhou, J, Li, Y: Existence and multiplicity of solutions for some Dirichlet problems with impulsive effects. Nonlinear Anal. 71, 2856-2865 (2009)

31. Rabinowitz, P: Minimax Methods in Critical Point Theory with Applications to Differential Equations. CBMS Regional Conference Series in Mathematics, vol. 65. Am. Math. Soc., Washington (1986)

32. Chang, K: Critical Point Theory and Its Applications. Modern Mathematics Series. Shanghai Scientific and Technology Press, Shanghai (1986)

33. Ekeland, I: Convexity Methods in Hamiltonian Mechanics. Springer, Berlin (1990)

34. Mawhin, J, Willem, M: Critical Point Theory and Hamiltonian Systems. Springer, Berlin (1989)

35. Simon, J: Régularité de la solution d'un problème aux limites non linéaires. Ann. Fac. Sci. Tolouse 3, 247-274 (1981)

Submit your manuscript to a SpringerOpen ${ }^{\mathcal{O}}$ journal and benefit from:

- Convenient online submission

- Rigorous peer review

Open access: articles freely available online

High visibility within the field

- Retaining the copyright to your article

Submit your next manuscript at $\boldsymbol{~ s p r i n g e r o p e n . c o m ~}$ 